\title{
Supporting Information - Bright Silicon Nanocrystals from a Liquid Precursor: Quasi-Direct Recombination with High Quantum Yield
}

\author{
Todd A. Pringle, ${ }^{* \dagger}$ Katharine I. Hunter,${ }^{\star}$ Alexandra Brumberg, ${ }^{\|}$Kenneth J. Anderson,, \\ Jeffrey A. Fagan, ${ }^{\#}$ Salim A. Thomas, ${ }^{\dagger}$ Reed J. Petersen, ${ }^{\S}$ Mahmud Sefannaser, ${ }^{\S}$ Yulun Han, ${ }^{\circledR}$ Samuel L. \\ Brown,$^{\dagger}$ Dmitri S. Kilin, ${ }^{\diamond}$ Richard D. Schaller,${ }^{\| \uparrow}$ Uwe R. Kortshagen,${ }^{\ddagger}$ \\ Philip Raymond Boudjouk, Erik K. Hobbie* ${ }^{\dagger, \S, \beta}$ \\ ${ }^{\dagger}$ Materials \& Nanotechnology Program, North Dakota State University, Fargo, North Dakota \\ 58108 \\ ${ }^{\star}$ Department of Mechanical Engineering, University of Minnesota, Minneapolis, Minnesota \\ 55455 \\ " Department of Chemistry, Northwestern University, Evanston, Illinois 60208 \\ ${ }^{\diamond}$ Department of Chemistry \& Biochemistry, North Dakota State University, Fargo, North Dakota \\ 58108 \\ \# National Institute of Standards \& Technology, Gaithersburg, Maryland 20899 \\ ${ }^{\S}$ Department of Physics, North Dakota State University, Fargo, North Dakota 58108 \\ ${ }^{\top}$ Center for Nanoscale Materials, Argonne National Laboratory, Argonne, Illinois 60439 \\ ${ }^{\beta}$ Department of Coatings \& Polymeric Materials, North Dakota State University, Fargo, North \\ Dakota 58108
}

DGU Process: SiNC size purification was performed in a Beckman Coulter Optima L-80 XP ultracentrifuge using a five-layer step gradient layered with $50 \%, 60 \%, 70 \%, 80 \%$, and $90 \%$ chloroform in m-xylene (by volume). All solvents were processed to remove oxygen. Custom polyvinylidene fluoride (Kynar) ultracentrifuge tubes were obtained from Seton Scientific. The step gradient was layered with a pipette, with subsequent layers deposited in order of decreasing density. A $0.3 \mathrm{~mL}$ solution of SiNCs in mesitylene (around $1 \mathrm{mg} / \mathrm{mL}$ ) was layered on top of the 
gradient. The layered tube was then immediately transferred to a SW-41 Ti swinging-bucket rotor and centrifuged for $12-20$ hours (depending on parent size) at $30,000 \mathrm{rpm}$ and $0{ }^{\circ} \mathrm{C}$. After the centrifuge run was complete, fractions were extracted in a nitrogen-filled glove box using a robotic pump system mounted on a micromanipulator.
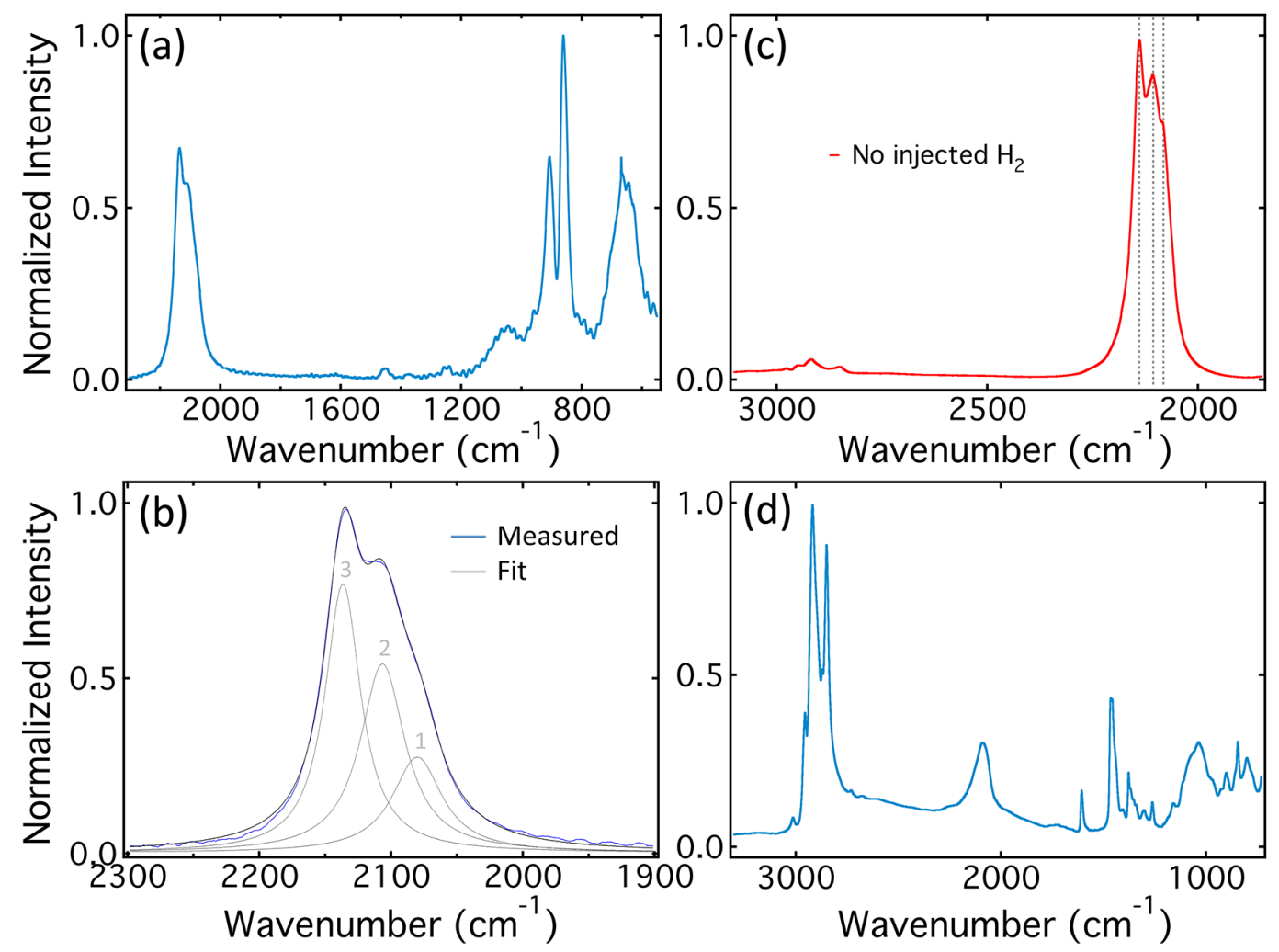

Figure S1. (a) FTIR spectrum of a dry reactant corresponding to a PL peak of $840 \mathrm{~nm}$ and QY of $60 \%$ post passivation. Data correspond to $46 \mathrm{sccm}$ carrier gas and $100 \mathrm{sccm}$ injected hydrogen. (b) Spectral analysis of the $\mathrm{SiH}_{x}$ peaks in panel (a) for $x=1,2$, and 3 as indicated. (c) FTIR spectrum of a dry reactant produced without injected hydrogen. Note that the $\mathrm{SiH}_{x}$ peaks are still present. The feature near $2900 \mathrm{~cm}^{-1}$ is attributed to extraneous hydrocarbons present in the glovebox used to process the sample and did not appear to correlate with QY. (d) FTIR spectrum of a dried colloid post-passivation, where the colloidal sample had a QY of $61 \%$ and a PL peak near $850 \mathrm{~nm}$. There is still unbound ligand in the sample (which was not "washed" with antisolvent) as evidenced by the small $\mathrm{C}=\mathrm{C}$ peak near $1650 \mathrm{~cm}^{-1}$. 


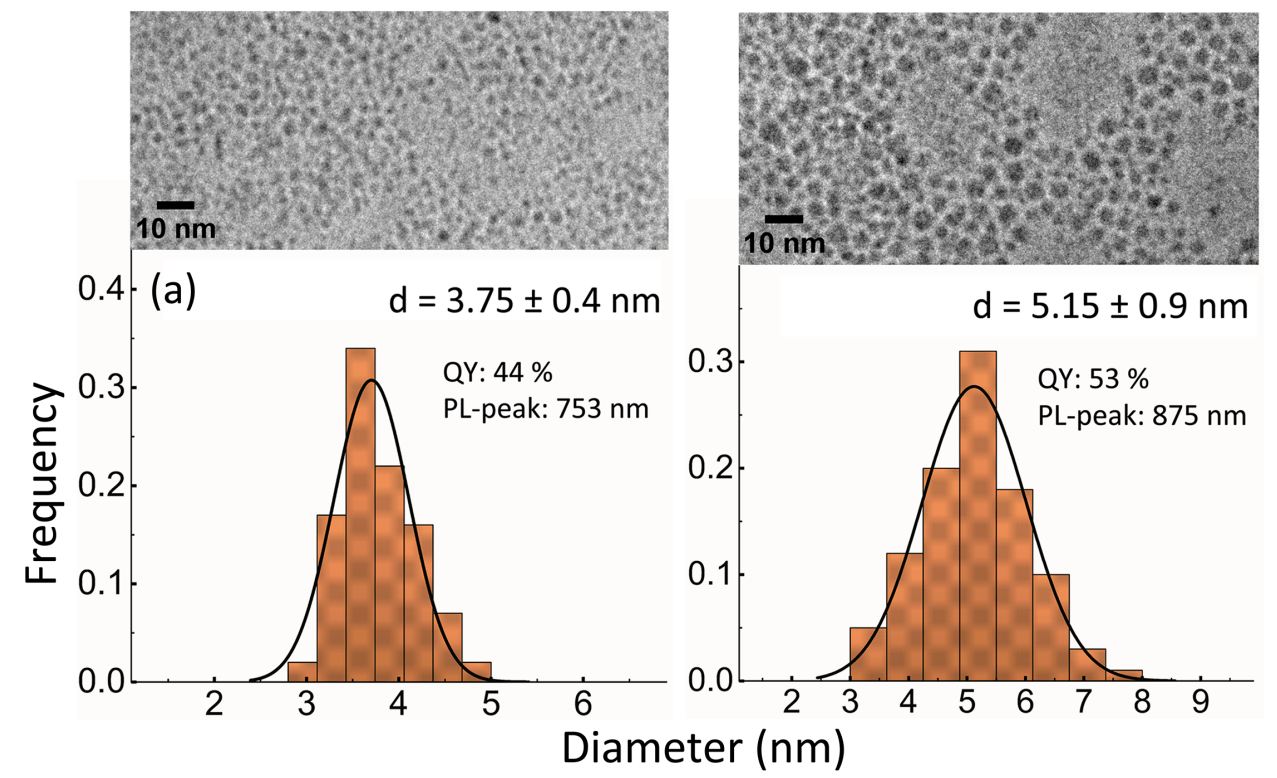

Figure S2. Representative TEM size distributions for as-produced colloidal SiNCs with mean size, quantum yield, and PL peak indicated.

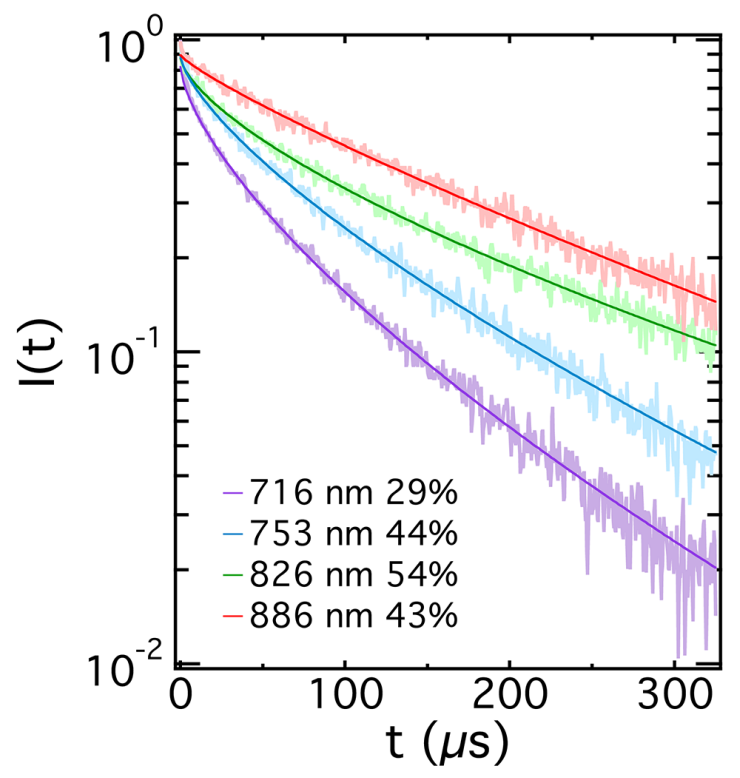

Figure S3. Representative slow PL relaxation measured for different parent SiNC colloidal suspensions. The solid curves are stretched exponential fits, with stretching exponents (top to bottom) of $0.85,0.67,0.71$, and 0.68 . PL QY for each sample is indicated in $\%$. 

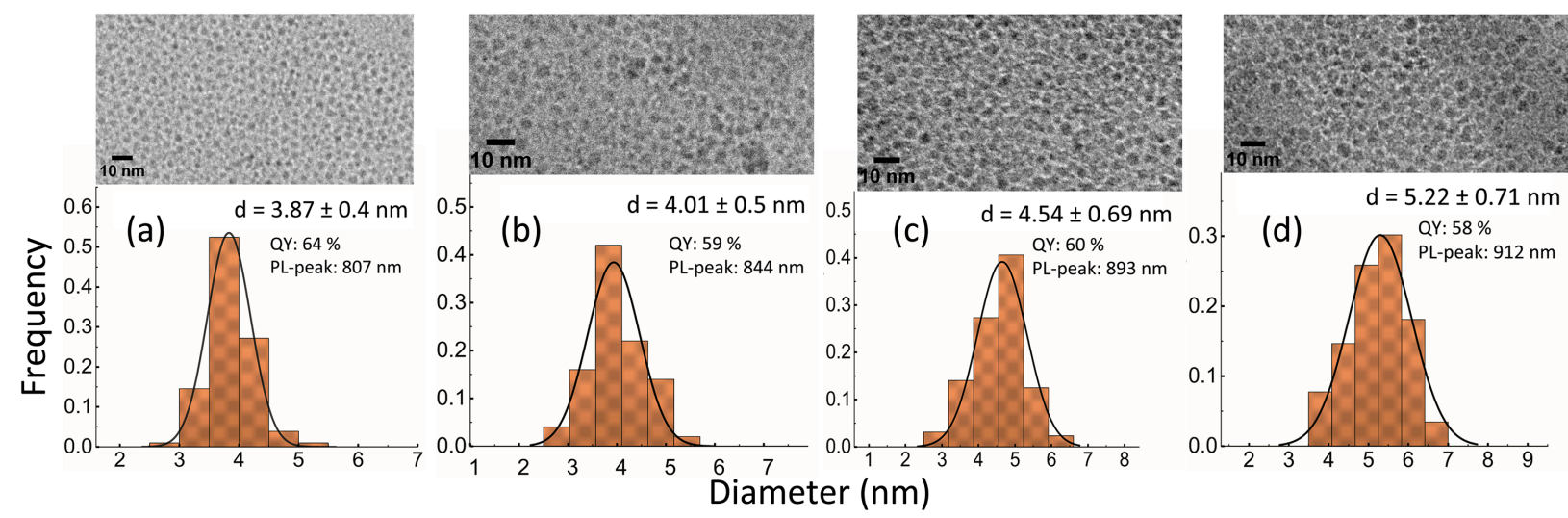

(d) $\begin{aligned} & \text { QY: } 58 \% \\ & \text { PL-peak: } 912 \mathrm{~nm}\end{aligned}$

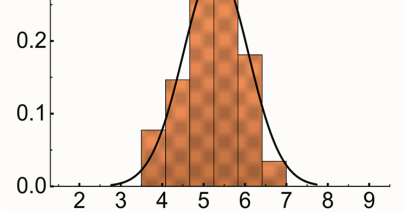

Figure S4. Representative TEM size distributions for size-resolved colloidal SiNC fractions with the mean size, distribution, quantum yield, and PL peak indicated.
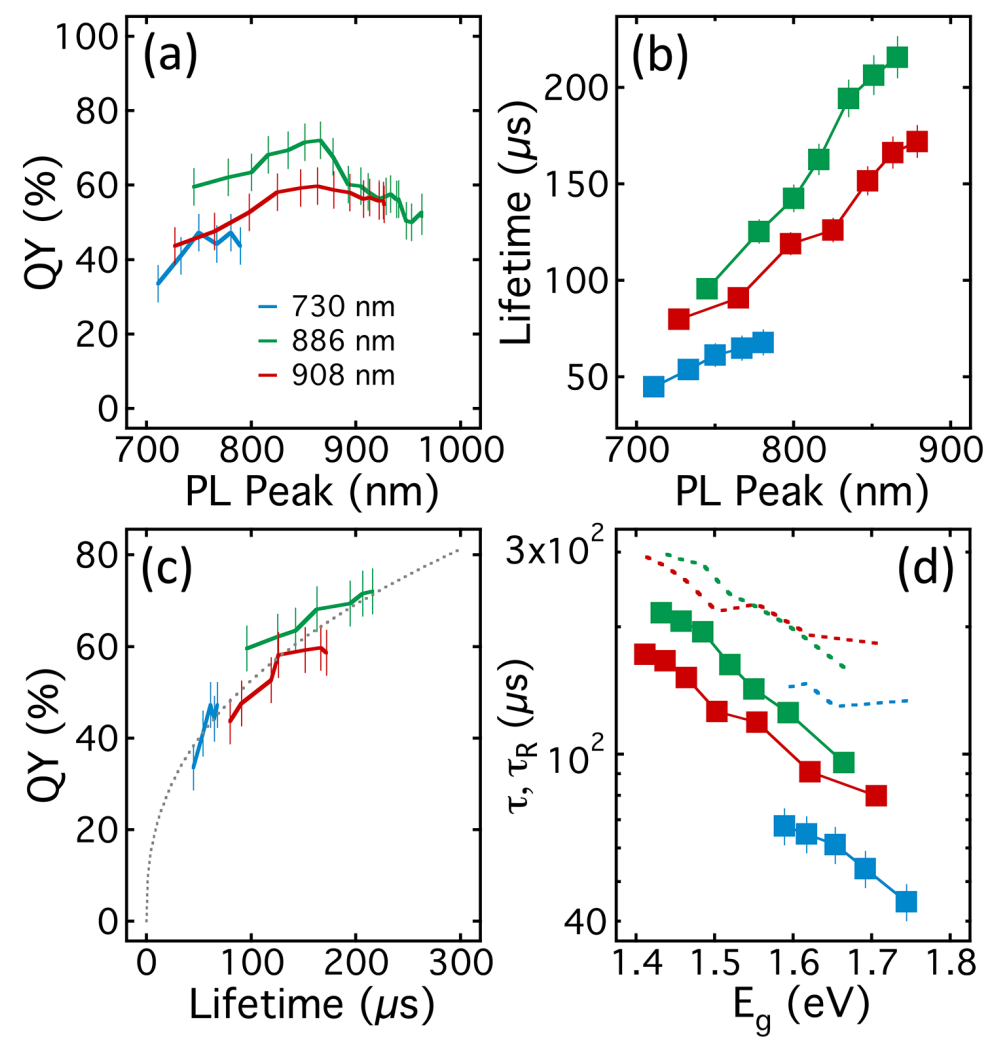

Figure S5. (a) QY versus PL peak for three sets of fractions extracted from three different parents of varied targeted size (parent PL peaks indicated). (b) Lifetime as a function of PL peak and (c) lifetime vs. QY for the same three sets of fractions. (d) Fraction lifetime and radiative lifetime (dashed, defined as $\tau$ divided by QY) as a function of PL peak energy for the same three sets of fractions. The color code in all panels corresponds to the legend in (a) and the curve in (c) 
is a guide to the eye. The curves in (c) are not expected to coincide given the variation in radiative lifetime with both fraction and parent.

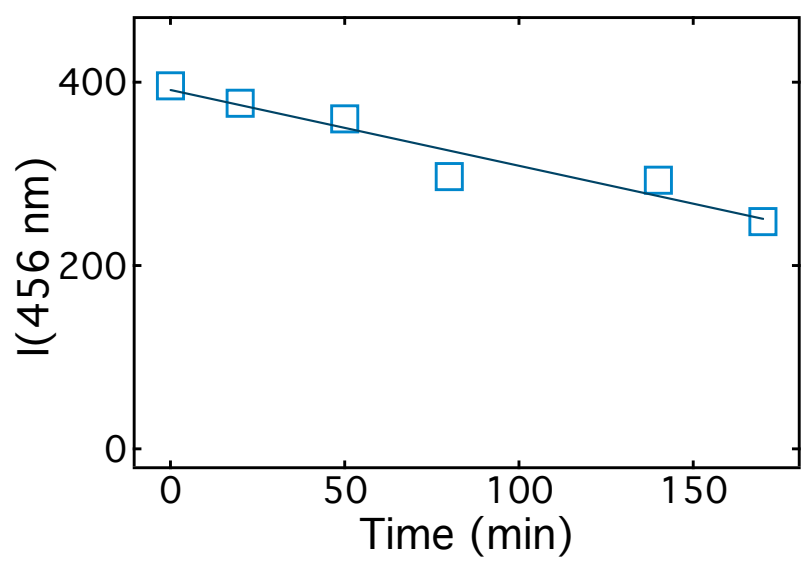

Figure S6. The effect of ambient oxygen exposure on the intensity of the fast PL mode for a sample with a PL peak of $834 \mathrm{~nm}$ and an initial QY of $65.7 \%$.

\begin{tabular}{|c|c|c|c|c|}
\hline PL Peak $(\mathrm{nm})$ & QY $( \pm 5 \%)$ & Bandgap $(\mathrm{eV})$ & ${\text { Diameter }(\mathrm{nm})^{\dagger}}^{\dagger}$ & $\tau(\mu \mathrm{s})$ \\
\hline 710 & 50 & 1.75 & 3.0 & $60 \pm 4.0^{*}$ \\
\hline 750 & 58 & 1.65 & 3.3 & $96 \pm 6.0$ \\
\hline 800 & 63 & 1.55 & 3.8 & $142 \pm 8.0$ \\
\hline 850 & 67 & 1.46 & 4.5 & $206 \pm 10.0$ \\
\hline 900 & 60 & 1.38 & 5.4 & $170 \pm 10.0$ \\
\hline 985 & 43 & 1.26 & 8.2 & ----- \\
\hline
\end{tabular}

Table S1. PL peak, QY, Bandgap, diameter and lifetime for a typical set of pristine fractions obtained from a high QY parent. 'Interpolated from the fit in Fig. 3e of the main text. "This value is extrapolated from a plot of lifetime versus bandgap.

Thermal Gravimetric Analysis (TGA): Ligand coverage was estimated using TGA applied to a parent SiNC suspension passivated with 1-octadecene, with a PL peak near $1060 \mathrm{~nm}$, a mean TEM diameter of $8.5 \mathrm{~nm}$, and a QY of $15 \%$. A larger nanocrystal/ligand pair was chosen to enhance TGA signal. The suspension was washed four times in a desktop centrifuge using sieved methanol as anti-solvent and sieved $\mathrm{N}_{2}$-sparged toluene as solvent. The purified colloid was thoroughly dried under vacuum to yield a dark brittle solid. For TGA, this was held at $100{ }^{\circ} \mathrm{C}$ 
under $\mathrm{N}_{2}$ for 30 min to remove impurities before initiating a $10 \mathrm{~K} / \mathrm{min}$ ramp in air up to $950{ }^{\circ} \mathrm{C}$, where the sample was held until the oxide mass stopped increasing. Assuming that all the remaining mass is $\mathrm{SiO}_{2}$, the final mass was converted to $\mathrm{Si}$ mass, which was then subtracted from the starting mass to get ligand mass. Converting each mass to moles, and using a ratio of surface to bulk Si atoms as given by $3 a / R$ where $a=0.235 \mathrm{~nm}$ is the nearest neighbor spacing of Si atoms, we get a surface ligand coverage of $40 \%$.
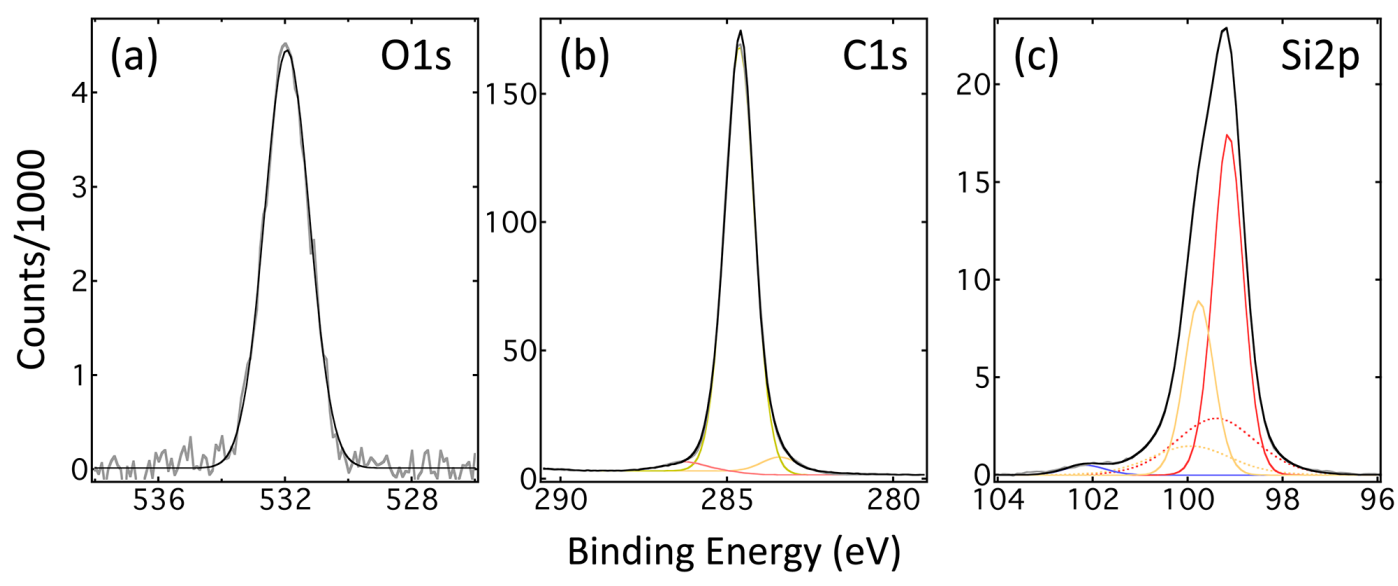

Figure S7. XPS analysis of a dried SiNC colloid (PL peak of $847 \mathrm{~nm}, \mathrm{QY}=60 \%$ ) for (a) the $1 s$ oxygen peak, (b) the $1 s$ carbon peak, and (c) the $2 p$ silicon peak. Data are gray and the fit is black. Colored curves show the underlying spectra used in the fit. In panel (c), blue indicates oxide, with oxygen being very weakly present in the interior of the sample [panel (a)]. The red and yellow curves are the Si $2 p_{1 / 2}$ and $2 p_{3 / 2}$ doublet near 100 and $99.5 \mathrm{eV}$, respectively, and the broad dashed curves under these can be interpreted as near surface silicon atoms. ${ }^{1}$

Computational Approach: A description of hot carriers in excited states requires explicit treatment of interactions between nuclear and electronic degrees of freedom. Here, electronphonon interaction is considered through the computation of "on-the-fly" nonadiabatic couplings from adiabatic molecular dynamics trajectories. ${ }^{2}$ The components of Redfield tensors in the density matrix formalism are then obtained by processing the autocorrelation function of 
nonadiabatic couplings and its Fourier transform. ${ }^{3-7}$ In this way the dissipative dynamics of the reduced density matrix is calculated.

Computation is performed on a $2.1 \mathrm{~nm}$ diameter SiNC surface passivated with hydrogen, with a chemical formula of $\mathrm{Si}_{220} \mathrm{H}_{144}$. The ground-state electronic structure is calculated with DFT in VASP using periodic boundary conditions, with $9 \AA$ of vacuum in each direction added to avoid spurious interactions between periodic images. ${ }^{8-12}$ The generalized gradient approximation $(\mathrm{GGA})^{13-14}$ with Perdew-Burke-Ernzerhof $(\mathrm{PBE})^{14}$ exchange-correlation functional in a plane-wave basis, along with projector augmented-wave (PAW) ${ }^{15}$ pseudopotentials, are used for DFT calculations. The plane-wave basis expansion has an energy cutoff of $500 \mathrm{eV}$.

Energies of Kohn-Sham orbitals and transition dipoles are extracted by processing the output of VASP DFT calculations. The absorbance spectrum $\alpha(\varepsilon)$ in the independent orbital approximation $(\mathrm{IOA})^{7}$ is

$$
\begin{gathered}
\alpha(\varepsilon)=\sum_{i j} f_{i j} \delta\left(\varepsilon-\Delta \varepsilon_{i j}\right) \\
\vec{D}_{i j}=\int \psi_{i}^{*} \vec{r} \psi_{j} d \vec{r} \\
f_{i j}=\left|\vec{D}_{i j}\right|^{2} \frac{4 \pi m_{e} \omega_{i j}}{3 \hbar e^{2}}
\end{gathered}
$$

where $f_{i j}$ is the oscillator strength between states $i$ and $j, \vec{D}_{i j}$ is the transition dipole moment, and $\omega_{i j}$ is the frequency of the incident light.

DFT-based adiabatic molecular dynamics calculations are performed at $300 \mathrm{~K}$ over a $1 \mathrm{ps}$ interval with a time step of $1 \mathrm{fs}$. The ab initio trajectories at ambient temperature allows for 
computing nonadiabatic couplings and components of the Redfield tensor. ${ }^{3-4}$ The approach to propagate hot carriers in excited states by combining on-the-fly nonadiabatic couplings with Redfield theory has been discussed in detail elsewhere. ${ }^{7,16-17}$ Here we briefly describe the methodology. Nonadiabatic couplings are computed according to the on-the-fly procedure along the adiabatic MD trajectory, ${ }^{2}$

$$
V_{i j}^{N A}(t)=-\frac{i \hbar}{2 \Delta t} \int d \vec{r} \varphi_{i}^{K S^{*}}\left(\vec{r},\left\{\vec{R}_{I}(t)\right\} \varphi_{j}^{K S}\left(\vec{r},\left\{\vec{R}_{I}(t+\Delta t)\right\}\right)+\right.\text { h.c. }
$$

Nonadiabatic couplings are then processed in terms of autocorrelation function averaging along the duration of the trajectory,

$$
M_{i j k l}(\tau)=\frac{1}{T} \int_{0}^{T} V_{i j}(t+\tau) V_{k l}(t) d t
$$

A Fourier transform of autocorrelation function provides components $\Gamma^{ \pm}$,

$$
\begin{gathered}
\Gamma_{i j k l}^{+}=\frac{1}{T} \int_{0}^{T} M_{i j k l}(\tau) e^{-i \omega_{k l} \tau} d \tau \\
\Gamma_{i j k l}^{-}=\frac{1}{T} \int_{0}^{T} M_{i j k l}(\tau) e^{-i \omega_{i j} \tau} d \tau
\end{gathered}
$$

These components provide Redfield tensors, which are averaged state-to-state transition rates,

$$
R_{i j k l}=\Gamma_{i j k l}^{+}+\Gamma_{i j k l}^{-}+\delta_{l j} \sum_{m} \Gamma_{i j k l}^{+}-\delta_{i k} \sum_{m} \Gamma_{i j k l}^{-}
$$

Components of the Redfield tensor control the dissipative dynamics of the density matrix,

$$
\left(\frac{d \rho_{i j}}{d t}\right)_{d i s s}=\sum_{l m} R_{j k l m} \rho_{l m}
$$


The evolution of electronic degrees of freedom can be described using Redfield theory in the density matrix formalism. The electronic degrees of freedom obey the equation of motion for the density operator $\hat{\rho}$, which can be cast in terms of the Liouville-von Neumann super-operator $\mathcal{L}$ and Redfield superoperator $\mathcal{R}$,

$$
\frac{d}{d t} \hat{\rho}=-(\mathcal{L}+\mathcal{R}) \hat{\rho}=\frac{\mathrm{i}}{\hbar}[\hat{\mathrm{F}}, \hat{\rho}]+\left(\frac{d \rho_{i j}}{d t}\right)_{d i s s}
$$

The nonequilibrium distribution of charge as a function of time and energy reads,

$$
n^{(a, b)}(\varepsilon, t)=\sum_{i} \rho_{i i}^{(a, b)}(t) \delta\left(\varepsilon-\varepsilon_{i}\right)
$$

where $(a, b)$ corresponds to the initial photoexcitation from state $a$ to state $b$.

The population change from the equilibrium distribution is,

$$
\Delta n^{(a, b)}(\varepsilon, t)=n^{(a, b)}(\varepsilon, t)-n^{e q}(\varepsilon, t)
$$

where $\Delta n>0$ describes population gain and $\Delta n<0$ corresponds to population loss.

The expectation value of a hot electron can be expressed as follows,

$$
\left\langle\Delta \varepsilon_{e}\right\rangle(t)=\sum_{i \geq L U} \rho_{i i}(t) \varepsilon_{i}(t)
$$

The above equation can be rewritten in terms of dimensionless energy,

$$
\left\langle E_{e}\right\rangle(t)=\frac{\left\langle\Delta \varepsilon_{e}\right\rangle(t)-\left\langle\Delta \varepsilon_{e}\right\rangle(\infty)}{\left\langle\Delta \varepsilon_{e}\right\rangle(0)-\left\langle\Delta \varepsilon_{e}\right\rangle(\infty)}
$$

The expectation values of a photoexcited hole are defined analogously.

The calculated populations of charge carriers allow for the calculation of exciton dynamics in the form of a distribution of energy as a function of excitation energy by, 


$$
P(\varepsilon, t)=\sum_{j \leq \mathrm{HO}} \sum_{i \geq \mathrm{LU}} \rho_{i i}(t) \rho_{j j}(t) \delta\left(\varepsilon_{i}^{e}-\varepsilon_{j}^{h}-\varepsilon\right)
$$

where $\varepsilon_{i}^{e}$ and $\varepsilon_{j}^{h}$ correspond to the energies of the populated electron and hole orbitals, respectively, $\rho_{j j}(t)$ and $\rho_{i i}(t)$ are the populations of two states, and $\delta$ is a Dirac delta function. The instantaneous time-resolved emission spectrum is obtained as,

$$
E(\hbar \omega, t)=\sum_{j>i} f_{i j} \delta\left(\hbar \omega-\hbar \omega_{i j}\right)\left\{\rho_{j j}(t)-\rho_{i i}(t)\right\}
$$

where $f_{i j}$ is the oscillator strength and $\hbar \omega_{i j}$ is the transition energy. The time-integrated emission spectrum is calculated as an integral of the time-resolved emission over the duration of the trajectory:

$$
E^{t o t}(\hbar \omega)=\frac{1}{T} \int_{0}^{T} E(\hbar \omega, t) d t
$$

\section{References}

1. Thiessen, A. N.; Ha, M.; Hooper, R. W.; Yu, H.; Oliynyk, A. O.; Veinot, G. C. G.; Michaelis, V. K. Silicon Nanoparticles: Are They Crystalline from the Core to the Surface? Chem. Mater. 2019, 31, 678-688.

2. Giustino, F. Electron-Phonon Interactions from First Principles. Rev. Mod. Phys. 2017, 89, 015003

3. Redfield, A. G. On the Theory of Relaxation Processes. IBM J. Res. Dev. 1957, 1, 19-31.

4. Jean, J. M.; Friesner, R. A.; Fleming, G. R. Application of a Multilevel Redfield Theory to Electron Transfer in Condensed Phases. J. Chem. Phys. 1992, 96, 5827-5842. 
5. Chen, J.; Schmitz, A.; Inerbaev, T.; Meng, Q.; Kilina, S.; Tretiak, S.; Kilin, D. S. FirstPrinciples Study of $P-N$-Doped Silicon Quantum Dots: Charge Transfer, Energy Dissipation, and Time-Resolved Emission. J. Phys. Chem. Lett. 2013, 4, 2906-2913.

6. Kilin, D. S.; Micha, D. A. Relaxation of Photoexcited Electrons at a Nanostructured Si(111) Surface. J. Phys. Chem. Lett. 2010, 1, 1073-1077.

7. Forde, A.; Inerbaev, T.; Hobbie, E. K.; Kilin, D. S. Excited-State Dynamics of a $\mathrm{CsPbBr}_{3}$ Nanocrystal Terminated with Binary Ligands: Sparse Density of States with Giant Spin-Orbit Coupling Suppresses Carrier Cooling. J. Am. Chem. Soc. 2019, 141, 4388-4397.

8. Kohn, W.; Sham, L. J. Self-Consistent Equations including Exchange and Correlation Effects. Phys. Rev. 1965, 140, A1133-A1138.

9. Kresse, G.; Hafner, J. Ab Initio Molecular Dynamics for Liquid Metals. Phys. Rev. B 1993, 47, $558-561$.

10. Kresse, G.; Hafner, J. Ab Initio Molecular-Dynamics Simulation of the Liquid-MetalAmorphous-Semiconductor Transition in Germanium. Phys. Rev. B 1994, 49, 14251-14269.

11. Kresse, G.; Furthmüller, J. Efficient Iterative Schemes for Ab Initio Total-Energy Calculations Using a Plane-Wave Basis Set. Phys. Rev. B 1996, 54, 11169-11186.

12. Kresse, G.; Furthmüller, J. Efficiency of Ab-Initio Total Energy Calculations for Metals and Semiconductors Using a Plane-Wave Basis Set. Comput. Mater. Sci. 1996, 6, 15-50.

13. Perdew, J. P.; Chevary, J. A.; Vosko, S. H.; Jackson, K. A.; Pederson, M. R.; Singh, D. J.; Fiolhais, C. Atoms, Molecules, Solids, and Surfaces: Applications of the Generalized Gradient Approximation for Exchange and Correlation. Phys. Rev. B 1992, 46, 6671-6687. 
14. Perdew, J. P.; Burke, K.; Ernzerhof, M. Generalized Gradient Approximation Made Simple. Phys. Rev. Lett. 1996, 77, 3865-3868.

15. Blöchl, P. E. Projector Augmented-Wave Method. Phys. Rev. B 1994, 50, 17953-17979.

16. Inerbaev, T. M.; Hoefelmeyer, J. D.; Kilin, D. S. Photoinduced Charge Transfer from Titania to Surface Doping Site. J. Phys. Chem. C 2013, 117, 9673-9692.

17. Han, Y.; Meng, Q.; Rasulev, B.; May, P. S.; Berry, M. T.; Kilin, D. S. Photoinduced Charge Transfer versus Fragmentation Pathways in Lanthanum Cyclopentadienyl Complexes. J. Chem. Theory Comput. 2017, 13, 4281-4296. 